\title{
ADESÃO AO USO DE MEDICAMENTOS: ALGUMAS CONSIDERAÇÕES
}

\author{
Carlos Alberto Mourão-Júnior \\ Professor Adjunto do Departamento de Fisiologia - Universidade Federal de Juiz de Fora.
}

André Bedendo de Souza

Graduando em Psicologia. Departamento de Psicologia - Universidade Federal de Juiz de Fora.

\begin{abstract}
Resumo
A adesão ao uso de medicamentos vem sendo estudada e discutida na literatura por inúmeros profissionais. Sua importância é crucial para o tratamento de um paciente e para a melhora do quadro de saúde do mesmo. O processo de adesão envolve muitas vezes uma ampla variedade de fatores. Dentre estes, pressupostos pessoais do paciente interferem diretamente na adesão. Assim, uma visão particular de cada paciente é fundamental para que se possa de fato compreender aquilo promove ou não a adesão à terapia. Este artigo tem por objetivo abordar variáveis envolvidas e estratégias para avaliar e promover a adesão, bem como promover reflexões que possam amadurecer esta discussão, principalmente no que se refere a perceber 0 paciente como fundamental no processo de adesão.
\end{abstract}

Palavras-chave: adesão à terapia, uso de medicamentos, tratamento medicamentoso.

\section{MEDICATION ADHERENCE: SOME CONSIDERATIONS}

\begin{abstract}
Medication compliance has been studied and discussed in the literature by many professionals. Its importance is crucial for the treatment of a patient and for the improvement of his health. The adherence process involves a wide variety of factors. Among these, the patient's personal assumption directly interferes in compliance. Thus, a particular vision of each patient is fundamental for the understanding of adherence or non adherence to therapy. This article aims to approach the variables involved and strategies to assess and promote adherence, as reflections that can ripen this discussion, especially as regards the patient as a crucial part of the adhesion process.
\end{abstract}

Keywords: compliance, adherence, medication.

\section{ADHESIÓN AL USO DE MEDICAMENTOS: ALGUNAS CONSIDERACIONES}

\begin{abstract}
Resumen
La adherencia al uso de medicamentos ha sido estudiada y discutida en la literatura por muchos profesionales. Su importancia es crucial para el tratamiento de un paciente y para la mejora de la salud de lo mismo. El proceso de adhesión implica una gran variedad de factores. Entre ellos, los supuestos personales del paciente interfieren directamente en la adhesión. Por lo tanto, una visión particular de cada paciente es fundamental para que podamos realmente entender lo que promueve o no adhesión al tratamiento. Este artículo tiene por objeto abordar las variables que intervienen y las estrategias para evaluar y promover la adhesión, así como las reflexiones que pueden madurar esta discusión, especialmente en lo que respecta a darse cuenta de la importancia que el paciente tiene en este proceso de adhesión.

Palabras clave: adhesión al tratamiento, uso de medicamentos, tratamiento medicamentoso.
\end{abstract}




\section{INTRODUÇÃO}

Apesar da adesão ao uso de medicamentos ter sido objeto de estudo de vários pesquisadores, trabalhos abordando esta temática são muito escassos na literatura brasileira. Estudos deste cunho são de fundamental importância para que se possa conhecer a relação entre paciente e medicamento e, consequentemente, permitir a redefinição de políticas públicas de saúde principalmente no que se refere ao Sistema Único de Saúde (SUS) -, possibilitando que novas estratégias sejam traçadas e que ocorram melhorias visando beneficiar os usuários do sistema.

Os gastos do Ministério da Saúde com medicamentos no ano de 2007 foram de mais de 4,5 bilhões de reais, o que representou 10,7 \% do valor gasto com medicamentos pelo Fundo Nacional de Saúde. Paralelamente ocorreu também um aumento significativo de $222 \%$ nas despesas com medicamentos entre os anos de 2002 e 2007 (Vieira, 2009). Estes valores retratam a importância dos gastos com fármacos no plano orçamentário nacional.

Medicamentos podem ser prescritos para se evitar maiores complicações na saúde de uma pessoa ou remissão de alguma doença, logo, presume-se que o não uso de medicações pode propiciar uma piora da mesma. Assim, pensando nos medicamentos como forma de se prevenir complicações futuras no estado de saúde de um indivíduo, caso uma pessoa deixe de utilizar fármacos receitados, ela se torna mais propensa a desenvolver complicações de saúde, e mais caro para o sistema público será para mantê-la em tratamento - pois, por exemplo, podem ocorrer internações decorrentes de complicações geradas pelo não cumprimento de uma terapia medicamentosa. Não obstante, um paciente aderente ao uso de medicações e que siga as recomendações de uso corretamente, tende a onerar menos do governo nos gastos com a saúde pública, pois evita que maiores danos à sua condição de saúde atual possam ocorrer. Consequentemente, nesta perspectiva, estudos sobre a adesão permitiriam que fossem evidenciadas razões que facilitariam o uso da medicação pela população, e posteriormente, estes poderiam servir de subsídio na reestruturação de políticas públicas relacionadas à dispensação de medicamentos.

De acordo com Cramer et al (2008), a adesão se refere ao ato de agir em conformidade com as recomendações dadas pelo cuidador, com relação ao tempo, à dosagem e à frequência de tomada de uma determinada medicação. 
Por conseguinte, a adesão é fator crucial no tratamento, pois permite que as recomendações feitas pelo profissional sejam realmente seguidas e consequentemente o objetivo final da intervenção terapêutica seja alcançado, ou seja, a melhora do paciente. No caso de uma terapia que envolva o uso de fármacos, o médico ao receitar determinado medicamento está somente iniciando um possível tratamento. Porém, para que este tratamento realmente se concretize, é necessário que o sujeito - alvo de tal medicamento -, faça o uso recomendado do mesmo, seguindo os critérios necessários para que efeitos desejáveis sejam alcançados.

Diante deste cenário, é natural alguém se perguntar: quais fatores estariam envolvidos neste processo de adesão? O que levaria uma pessoa a usar ou não o medicamento prescrito? O que faz com que um paciente siga seu tratamento até o fim? Este artigo tem por objetivo abordar tais questões e expor pontos importantes referentes à adesão ao uso de medicamentos, possibilitando que trabalhos futuros possam amadurecer esta discussão e propor novas estratégias de ação.

Variáveis envolvidas no processo de adesão

A discussão sobre a adesão a medicamentos envolve inúmeros fatores que se relacionam de maneira ampla e complexa. No entanto, no âmbito científico nacional são necessárias mais investigações acerca de variáveis que estão envolvidas e relacionadas com a adesão, a fim de melhor se compreender fatores que de realmente facilitam ou não a mesma. Não existe uma variável única que faça um indivíduo aderir às recomendações feitas por um profissional em relação a quais remédios utilizar. A não adesão está envolvida com fatores biológicos, psicológicos, culturais e comportamentais, que se inter-relacionam. Estes fatores subsidiam as decisões de um indivíduo sobre usar ou praticar aquilo que the foi indicado. Assim, neste trabalho vamos explorar as seguintes variáveis: tempo, cultura, número de medicamentos utilizados, associação de fármacos em um único medicamento, concordância entre médico-paciente e distribuição gratuita de medicamentos.

\section{Tempo}

Não há definição quanto ao tempo que um indivíduo deve permanecer utilizando determinada medicação para ser considerado aderente. Pesquisas na 
área utilizam-se de períodos variáveis de tempo para indicar se o paciente cumpriu as orientações médicas em relação ao uso dos medicamentos e, consequentemente, aderiu à terapia. Estes períodos podem variar de poucos dias a alguns meses após a prescrição dos medicamentos.

Existem diversos tipos de medicamentos, os quais possuem características farmacológicas distintas. Logo, suas ações no organismo podem ser mais lentas ou rápidas. Por exemplo, no caso de um analgésico comum comprado em farmácias, é esperado que sua ação se dê em cerca de 30 minutos após ser ingerido. Contudo, um antidepressivo, por sua vez, possui ação esperada após um uso de duas semanas. Assim, é provável que indivíduos que não tenham sido informados sobre estas condições, deixem de utilizar a medicação por não perceberem mudanças em seus sintomas imediatamente após o início do tratamento.

\section{Cultura}

Sabe-se que cada população possui características culturais próprias e que influenciam diretamente a maneira com que se relaciona com o mundo. Desta forma, a cultura de uma sociedade também pode agir sobre os padrões de consumo de medicamentos da mesma. Isto é evidente em países com ampla área territorial e grande diversidade cultural como o Brasil, onde diferenças regionais entre a maneira de a população utilizar medicamentos podem ser percebidas. Estudos demonstram que a prevalência do uso de medicamentos pela população idosa de Belo Horizonte, Minas Gerais, é de 72,1\% (Loyola Filho, Uchoa \& Lima-Costa, 2006) e de 91\% em idosos de Porto Alegre, Rio Grande do Sul (Flores \& Mengue, 2005). Com isto, a fim de melhor se compreender as razões que levam determinado grupo a ser aderente ou não à terapia, é fundamental que se leve em consideração as características culturais da população envolvida.

\section{Número de medicamentos utilizados}

Uma variável que vem sendo amplamente investigada em relação à adesão à terapia medicamentosa é o número de medicamentos utilizados (Brekke, Hunskaar \& Straand, 2006; Russel, Vicki \& Jantarakupt, 2006; Balkrishnan, 1998; Leite \& Vasconselos, 2003). O número médio de medicamentos usados varia de acordo com a população estudada. Estudos brasileiros descrevem desde 
1,3 em idosos residentes nas áreas periféricas da cidade de Fortaleza (Coelho Filho, Marcopito \& Castelo, 2004) até 3,2 medicamentos em idosos da cidade de Porto Alegre (Flores \& Mengue, 2005).

O estudo desta variável é importante pois quanto maior o número de medicamentos prescritos para um paciente idoso, menor é sua adesão (Coons et al, 1994). Além disto, quanto mais medicamentos utilizados, maior é o risco de internações durante a não adesão (Col, Fanale \& Kronholm, 1990). Assim, o médico que prescreve remédios a seu paciente deve estar informado de que maiores quantidades de medicação tendem a propiciar menores chances de adesão deste último, além de possíveis complicações decorrentes da mesma.

\section{Associação de fármacos em um único medicamento}

Para evitar que a adesão não ocorra devido ao uso de muitos medicamentos, uma alternativa seria a prescrição de uma associação de fármacos em uma mesma dose terapêutica, ou seja, em um único comprimido, por exemplo. Isto seria viável por seu caráter de utilização facilitada e custos reduzidos. Contudo, Rozenfeld (2003) ressalta que esta prática é desaconselhável pelo fato de serem potencializadas as chances de ocorrerem reações adversas, além de ser mais difícil a discriminação da substância que tenha causado tais reações.

Neste contexto a cautela do profissional ao prescrever este tipo de medicamento deve ser redobrada, a fim de julgar os benefícios e possíveis riscos de sua indicação.

\section{Concordância entre médico e paciente}

Outro ponto de fundamental importância para a adesão à terapêutica medicamentosa prescrita refere-se à relação entre médico e paciente. Foi demonstrado que pacientes com altos níveis de concordância, ou consentimento, com seu médico foram um terço mais aderentes ao uso das medicações prescritas durante uma consulta (Kerse et al, 2004). Neste contexto, é perceptível a importância do médico no momento em que prescreve alguma medicação, pois certas condutas adotadas por ele podem promover determinadas condições que favoreçam ou não o uso do medicamento. Com isto, o médico deve fomentar a idéia de que são importantes as recomendações feitas por ele, para que o paciente possivelmente concorde com a necessidade do uso 
da medicação, e, assim, ao deixar o consultório, siga com as orientações prescritas. Nesta perspectiva, o médico deve assumir uma postura amigável e que transmita confiança ao paciente, potencializando assim as chances deste último seguir as recomendações feitas.

\section{Distribuição gratuita de medicamentos}

A condição econômica da população também possui relação com o uso de medicamentos. No Brasil, é bastante claro que diversos indivíduos não possuem condições financeiras para suprir todos seus gastos com medicações. A distribuição gratuita de medicamentos é fundamental neste contexto para que as classes mais pobres tenham acesso aos fármacos de que necessitam. Para permitir que de alguma maneira isto ocorra, no Brasil existe a distribuição de medicamentos básicos pelo governo federal. Contudo, nem todas as medicações existem em quantidades e variedade suficientes para suprir completamente as necessidades da população.

Goldman, Joyce e Zheng (2007) relatam que quanto maior é o custo da medicação ao usuário final, ou seja, ao paciente, maior é sua associação com uma pior adesão e mais freqüente é a descontinuação da terapia. Estes autores propõem como estratégia para contornar este problema, o aumento dos benefícios farmacêuticos, julgando-os como uma importante ferramenta de saúde pública para a redução dos custos da medicação. Portanto, na medida em que se permite à população maiores chances de adquirir o medicamento de que necessita, maior tende a ser a adesão da mesma.

\section{Estratégias para avaliar a adesão e Métodos}

Atualmente existem diversas estratégias para se investigar a adesão ao uso de medicamentos. Tais formas de avaliação variam de técnicas mais sofisticadas às maneiras mais simples e de menor custo. No entanto ainda não existe uma que seja considerada padrão-ouro.

Boudes (1998) destaca métodos para avaliar adesão, tais como: entrevistas, contagem de pílulas, dispositivos eletrônicos de controle de doses, diário do paciente com dados sobre o uso da medicação, e, coleta de material biológico para mensuração de doses. Alguns destes métodos são mais acurados que outros, porém tendem a ser mais caros e de mais difícil utilização - como, por exemplo, a coleta de sangue para aferir concentrações da medicação no 
organismo. Outros métodos, por sua vez, são mais baratos e simples - como entrevistas ao paciente durante a consulta. Porém, estas tendem a superestimar as respostas e devem ser utilizadas como complemento a outros métodos de avaliação.

Cabe destacar que estas estratégias podem ser utilizadas de maneira conjunta, a fim de melhor investigar uma possível não adesão à terapia, para que, assim, com mais subsídios, possa-se identificar quais fatores estão dificultando a adesão do paciente.

Ainda existe a necessidade de se obter um instrumento que possa medir a adesão a um tratamento e que seja minimamente invasivo para o paciente (Cramer, Mattson, Prevey, Scheyer \& Oullette, 1989). Com um instrumento de monitoramente contínuo e mais preciso, dados mais válidos e fidedignos que não superestimem nem subestimem a realidade estudada poderiam ser acessados, e, por conseguinte, estratégias mais precisas para promover a adesão poderiam ser utilizadas.

\section{Foco no paciente}

O paciente é ponto principal na adesão a uma terapia, e por isto deve ser percebido como tal. Sua identidade, necessidades e condições devem ser reconhecidas e consideradas. Ele é quem decide aderir a um tratamento e somente o fará a partir do momento em que aceitar como importante para si a terapia proposta. Assim, a partir desta perspectiva, os profissionais que orientam o uso de medicamentos devem fornecer aos pacientes todas as informações necessárias a fim de que os mesmos possam perceber como importante o uso da medicação prescrita na mudança de seu estado de saúde atual.

Umas das maneiras de se focar o paciente é atentar-se às razões que de fato importam para cada indivíduo e que permitem que ele siga a terapia medicamentosa proposta. Conrad (1985) percebeu em seu estudo que o uso do medicamento era favorecido pela capacidade do fármaco de minimizar a estigmatização causada pela condição médica e assegurar certa 'normalidade" evitar situações que não sejam comuns em indivíduos sem determinada condição médica, por exemplo, convulsões. Isto demonstra que subjazem questões internas ao indivíduo e que podem promover ou não a adesão. Ou seja, existem certas questões individuais e particulares que são levadas em conta no momento de escolha entre seguir ou não as orientações médicas. Assim, atentando-se ao 
sujeito se poderá compreender melhor o porquê de uma pessoa seguir de fato o que Ihe foi recomendado, bem como intervir para que ela possa aderir melhor ao tratamento.

\section{Estratégias para promover adesão e formas de intervenção}

As pessoas tendem a manter-se em uma zona de conforto, e mudanças que interfiram na mesma inclinam-se a ser percebidas como desagradáveis. Seria como se ocorresse uma perda de equilíbrio e sua segurança estivesse ameaçada. Cabe então ao profissional, médico ou não, ajudar cada sujeito a sair desta condição, proporcionando a ele a segurança necessária para tal. Isto só se torna possível quando se atenta para o próprio sujeito e compreende-se que ele possui particularidades que o levam a agir de determinada maneira.

Seguindo esta perspectiva de tentar promover a adesão, algumas estratégias podem ser utilizadas na tentativa de ampliar as chances de um indivíduo ser mais aderente a uma terapia medicamentosa.

Existem algumas maneiras as quais o profissional pode utilizar para promover a adesão. Tais estratégias podem ser através de: instrução oral e escrita; orientação terapêutica ao paciente e à família; utilização das respostas do próprio organismo do paciente como alterações de pressão arterial, convulsões - que alertam para melhoras ou pioras decorrentes do uso ou não do medicamento -; lembretes; e recompensas (McDonald, Garg \& Haynes, 2002). Desta forma, ao utilizar, por exemplo, alterações de pressão arterial e convulsões que o paciente pode ter apresentado devido ao mau ou não uso de medicamentos, o profissional permite que o próprio sujeito perceba as consequências de sua não adesão, e possivelmente possa modificar sua atitude a fim de evitar novas ocorrências de condições desagradáveis.

Roter et al (1998) acrescentam que a combinação entre componentes afetivos, comportamentais e cognitivos com intervenções compreensivas são mais efetivas do que intervenções de foco único. Por sua vez, Rich, Gray, Beckham, Wittenberg \& Luther (1996), argumentam que uma estratégia de tratamento multidisciplinar está associada a um aumento na adesão de medicamentos em um período de trinta dias após alta hospitalar em pacientes idosos com problemas cardíacos.

No intuito de promover condições que criem ao paciente uma atmosfera em que ele sinta mais segurança e obtenha mais conhecimento sobre sua condição, 
pode ser utilizada uma técnica conhecida como psicoeducação. Ela propõe a promoção de conhecimento, visando favorecer uma conduta mais aderente do indivíduo à terapia. Questões relativas a como o medicamento age no organismo, efeitos esperados do mesmo e o tempo que ele leva para proporcionar uma melhora do quadro podem ser discutidos, buscando o aprendizado do paciente. $A$ partir daí, inseguranças e medos dos indivíduos podem ser diminuídos e consequentemente a adesão poderá ser promovida, evitando-se assim falsas expectativas sobre o uso da medicação.

\section{Avaliação específica do caso}

Existem algumas estratégias relatadas para se promover a adesão, contudo não existe uma que seja considerada ideal e deva sempre ser utilizada. A avaliação específica do caso do paciente por parte do profissional pode identificar possíveis razões pessoais da não adesão daquele indivíduo. Por exemplo, duas pessoas que possuem respectivamente, condição financeira restrita e receio das reações que o medicamento pode lhe causar, podem optar por não seguir a terapia medicamentosa. Assim, motivos diferenciados podem culminar em um mesmo resultado: a menor adesão. A partir daí, uma intervenção mais focada naquela situação poderia ser considerada, e, consequentemente, a efetividade desta seria maximizada, assim como possivelmente a da terapia. Isto porque a intervenção fora escolhida para aquele caso em particular, levando em consideração as peculiaridades do mesmo.

\section{CONSIDERAÇÕES FINAIS}

A temática da adesão ao uso de medicamentos é de fundamental importância na terapêutica do paciente. O conhecimento dos fatores que levam ao uso ou não de fármacos prescritos por um profissional permite a criação de melhores maneiras de demonstrar ao paciente a real importância de determinado tratamento na mudança da sua condição atual. Além disto, problemas decorrentes de um uso incorreto, ou, até mesmo o não uso dos medicamentos no tratamento de uma condição clínica podem ser evitados. Desse modo, para que a adesão ocorra é de crucial importância que o profissional que prescreva ou estimule o uso de uma medicação assuma uma postura na qual a escuta e a comunicação sejam percebidas como pontos chave. Somente um profissional que 
consiga ouvir e entender corretamente seu paciente, também conseguirá ser ouvido e compreendido, e, possivelmente, ter suas orientações seguidas.

\section{REFERÊNCIAS}

Balkrishnan, R. (1998). Predictor of medication adherence in the elderly. Clinical Therapeutics, 20(4), 764-771.

Boudes, P. (1998). Drug compliance in therapeutic trials: a review. Controlled Clinical Trials, 19(3), 257-268.

Brekke, M., Hunskaar, S., \& Straand, J. (2006). Self-reported drug utilization, health, and lifestyle factors among 70-74 year old community dwelling individuals in Western Norway. The Hordaland Health Study (HUSK). BMC Public Health, 6(121).

Coelho Filho, J. M. C., Marcopito, L. F., \& Castelo, A. (2004). Perfil de utilização de medicamentos por idosos em área urbana do nordeste do Brasil. Revista de Saúde Pública, 38(4), 557-564.

Col, N., Fanale, J. E., \& Kronholm, P. (1990). The role of medication noncompliance and adverse drug reactions in hospitalizations of the elderly. Archives of Internal Medicine, 150(4), 841-845.

Conrad, P. (1985). The meaning of medication: another look at compliance. Social Science and Medicine, 20(1), 29-37.

Coons, S. J., Sheahan, S. L., Martin, S. S., Hendricks, J., Robbins, C.A., \& Johnson, J.A. (1994). Predictors of medication noncompliance in a sample of older adults. Clinical Therapeutics, 16(1), 110-117.

Cramer, J. A., Mattson, R. H., Prevey, M. L., Scheyer, R. D., \& Oullette, V. L. (1989). How often is medication taken as prescribed? A novel assessment technique. JAMA, 261(22), 3273-3277.

Cramer, J. A., Roy, A., Burrell, A., Fairchild, C. J., Fuldeore, M. J., Ollendorf, D. A., \& Wong, P. K. (2008). Medication compliance and persistence: terminology and definitions. Value in Health 2(1), 44-47.

Flores, L. M., \& Mengue, S. S. (2005). Uso de medicamentos por idosos em região do sul do Brasil. Revista de Saúde Pública, 39(6), 924-929.

Goldman, D. P., Joyce, G. F., \& Zheng, Y. (2007). Prescription drug cost sharing: Association with medication and medical utilization and spending health. JAMA, 298(1), 61-69. 
Kerse, N., Buetow, S., Mainous III, A. G., Young, G., Coster, G., \& Arroll, B., (2004). Physician-patient relationship and medication compliance: a primary care investigation. Annals of Family Medicine, 2(5), 455-461.

Leite, S.N., \& Vasconselos, M.P.C. (2003). Adesão à terapêutica medicamentosa: elementos para a discussão de conceitos e pressupostos adotados na literatura. Ciência e Saúde Coletiva, 8(3), 775-782.

Loyola Filho, A. I. L., Uchoa, E., \& Lima-Costa, M. F., (2006). Estudo epidemiológico de base populacional sobre uso de medicamentos entre idosos na região metropolitana de Belo Horizonte, Minas Gerais, Brasil. Caderno de Saúde Pública, 22(12), 2657-2667.

Marques, M. B. (2000), Patentes farmacêuticas e acessibilidade aos medicamentos no Brasil'. História, Ciências, Saúde - Manguinhos, 7(1), 721.

McDonald, H. P., Garg, A. X., \& Haynes, R. B. H. (2002). Interventions to enhance patient adherence to medication prescriptions. JAMA, 288(22), 2868-2879.

Rich, M., Gray, D.B., Beckham, V., Wittenberg, C., \& Luther, P. (1996) Effect of a multidisciplinary intervention on medication compliance in elderly patients with Congestive heart failure. The American Journal of Medicine, 101(3), 270-276.

Roter, D. L., Hall, J. A., Merisca, R., Nordstrom, B., Cretin, D., \& Svarstad, B. (1998) Effectiveness of interventions to improve patient compliance: a meta-analysis. Medical Care, 36(8), 1138-1161.

Rozenfeld, S. (2003). Prevalência, fatores associados e mau uso de medicamentos entre os idosos: uma revisão. Caderno de Saúde Pública, 19(3), 717-724.

Russel. C. L., Vicki, S. C., \& Jantarakupt, P. (2006). Older adult medication compliance: integrated review of randomized controlled trials. American Journal of Health Behavior, 30(6), 636-650.

Vieira, F. S. (2009). Gasto do Ministério da Saúde com medicamentos: tendência dos programas de 2002 a 2007. Revista de Saúde Pública, 43(4), 674-981. 
Contato: depto.fsi@ufjf.edu.br

Recebido em: 01/02/2010

Revisado em: 20/04/2010

Aceito em: 15/06/2010 